\title{
Stress testing and myocardial perfusion imaging for patients after recovery from severe COVID- 19 infection requiring hospitalization: A single- center experience
}

\author{
Usman A. Hasnie, MD, ${ }^{\text {a }}$ Riem Hawi, MD, , c, Efstathia Andrikopoulou, MD, ${ }^{b}$ \\ Ami E. Iskandrian, MD, MASNC, ${ }^{\mathrm{b}}$ and Fadi G. Hage, MD, MASNC ${ }^{\mathrm{b}, \mathrm{c}}$ \\ a Department of Medicine, University of Alabama at Birmingham, Birmingham, AL \\ b Division of Cardiovascular Disease, Department of Medicine, University of Alabama at \\ Birmingham, Birmingham, AL \\ c Section of Cardiology, Birmingham Veterans Affairs Medical Center, Birmingham, AL
}

Received Jan 16, 2021; accepted Mar 8, 2021

doi: $10.1007 / \mathrm{s} 12350-021-02606-w$

Background. As the coronavirus pandemic progresses, patients that have recovered from COVID-19-related hospitalization require resumption of care for other medical issues. Thus far, the literature has not detailed the experience of stress testing in this patient population.

Methods. We retrospectively reviewed patients that recovered from COVID-19-related hospitalizations and underwent SPECT MPI studies at the University of Alabama at Birmingham Medical Center.

Results. 15 patients (median age 60 years, $67 \%$ male) were identified with COVID-19related hospitalization and then underwent SPECT MPI imaging after recovery. During COVID-19-related hospitalization (median length of stay 8 days), patients received various COVID-19 therapies; 3 required mechanical ventilation. Stress tests (4 Exercise, 11 Pharmacologic) were performed 65 days (interquartile range 31-94 days) after the diagnosis of COVID19. None of the patients experienced serious adverse events during or after stress testing. One patient required regadenoson reversal using aminophylline due to chest pain.

Conclusion. Over time, more patients that recover from COVID-19 infection will require MPI testing for myocardial ischemia evaluation. Our study provides some information regarding performing stress testing in patients who have recently recovered from COVID-19 infections requiring hospitalization. Further studies are recommended to establish formal protocols for testing in this cohort. (J Nucl Cardiol 2021;28:2167-73.)

Key Words: CAD • SPECT • vasodilators • exercise testing

The authors of this article have provided a PowerPoint file, available for download at SpringerLink, which summarizes the contents of the paper and is free for reuse at meetings and presentations. Search for the article DOI on SpringerLink.com.

All editorial decisions for this article, including selection of reviewers and the final decision, were made by guest editor Nagara Tamaki, MD.

Dr. Hage reports investigator-initiated grant support from Astellas Pharma.
Supplementary Information The online version contains supplementary material available at https://doi.org/10.1007/s12350-02102606-w.

Reprint requests: Usman A. Hasnie, MD, Department of Medicine, University of Alabama at Birmingham, Birmingham, AL ; uhasnie@uabmc.edu

J Nucl Cardiol 2021;28:2167-73.

1071-3581/\$34.00

Copyright $\Subset 2021$ American Society of Nuclear Cardiology. 


\section{INTRODUCTION}

During the peak of the pandemic, in an attempt to preserve personal protective equipment while protecting patients and healthcare workers, there was a marked reduction in cardiovascular imaging studies and procedures including stress tests using singlephoton emission computed tomography (SPECT) myocardial perfusion imaging (MPI). ${ }^{1-5}$ The American Society of Nuclear Cardiology (ASNC) provided guidance on how best to navigate the initial phase of the pandemic when non-emergent evaluations were postponed and thereafter when nuclear cardiology laboratories began to resume testing. ${ }^{6,7}$ Now, as the pandemic progresses, providers are encountering patients that have recovered from COVID-19 infections and require MPI for multiple indications. Since recovery from COVID-19 is variable with many patients having persistent shortness of breath, reduced quality of life and fatigue for weeks, perceived inability to tolerate MPI can present a dilemma to providers. $^{8}$ In this manuscript, we report on the initial data from a single institution regarding our experience in performing MPI in patients that recovered from severe COVID-19-related hospitalizations.

\section{METHODS}

We retrospectively identified patients at the University of Alabama at Birmingham who underwent stress MPI for all indications after recovering from COVID-19-related hospitalization from March to October 2020. Patient demographics, past medical history, indication for and findings on MPI were obtained from medical records.

Standard ASNC protocols were used for MPI testing. ${ }^{9}$ Performance and interpretation of MPI at our institution has been described previously. ${ }^{10-14}$ A stress-first protocol was used. Patients were provided a mask to wear during testing. Although we purposefully decreased the performance of exercise stress tests during the pandemic, when personal protective equipment (PPE) supplies were available, our institution offered exercise MPI in select patients that tested negative for COVID-19 using polymerase chain reaction within 72 hours prior to undergoing MPI while quarantining. Staff in the stress room were fitted with proper PPE including N95 masks, gowns, gloves, hair caps, and face shields.

We observed for the occurrence of serious complications during or following stress testing and for symptoms reported during the test. As summary statistics, the median [interquartile range] of continuous data and the frequency (percentage) of categorical data are shown.

\section{RESULTS}

During the study period, 15 patients underwent stress testing with MPI at our institution after recovering from COVID-19-related hospitalization. Summary statistics are shown in Table 1 and data for the individual patients are shown in Appendix 1A-B. The median age of the cohort was 60 years [51-68] and more than half of the patients were Black. There was a high prevalence of risk factors and comorbidities, but none of the patients had a prior history of myocardial infarction. The vast majority of COVID hospitalizations (median length of stay 8 days) were related to respiratory distress or failure. Almost half of the patients required intensivecare-unit-level care during their hospitalization. Of the three patients that required mechanical ventilation, this was maintained for 16,31 , and 33 days, respectively. Patients received varied therapies for COVID-19 infection ranging from supportive care to dexamethasone/ remdesivir as well as investigational therapies as part of clinical trials.

The majority of the stress tests were completed in the outpatient setting and were performed for evaluation of chest pain or shortness of breath (Table 1). The median duration between COVID-19 diagnosis and MPI was 65 days (earliest at 22 days). Two patients had MPI prior to discharge from their COVID-related hospitalization. Most patients (80\%) had a negative COVID-19 test prior to undergoing stress testing with most of these occurring within 72 hours of MPI. Most of the studies were performed using regadenoson rather than exercise. All the exercise studies were terminated due to fatigue after achieving on average $95 \%$ of maximal agepredicted heart rate. The majority of the patients had normal perfusion with one (7\%) demonstrating scar in the distribution of the left anterior descending artery. The average LVEF was $55 \%$.

None of the patients had serious adverse events after stress testing including no death, cardiac or respiratory arrest, myocardial infarction, stroke, hospitalization, significant arrhythmias (persistent or hemodynamically significant supraventricular or ventricular tachycardia, ventricular fibrillation, high-grade atrioventricular block, or asystole), seizures, or severe bronchospasm. Of the 11 patients who underwent pharmacologic stress, one patient reported chest pain after regadenoson administration and more than half had dyspnea, but these symptoms were not severe. The patient that experienced chest pain received aminophylline, but this was administered more than 2 minutes 
Table 1. Baseline demographics, MPI study qualitative data

\begin{tabular}{|c|c|}
\hline \multicolumn{2}{|c|}{ Demographics } \\
\hline Age & $\begin{array}{l}60 \text { years [51- } \\
68]\end{array}$ \\
\hline Male gender & $10(66.7 \%)$ \\
\hline \multicolumn{2}{|l|}{ Race } \\
\hline Caucasian & $5(33.3 \%)$ \\
\hline Black & $8(53.3 \%)$ \\
\hline Other & $2(13.3 \%)$ \\
\hline Diabetes & 7 (46.7\%) \\
\hline Hypertension & $11(73.3 \%)$ \\
\hline Dyslipidemia & 7 (46.7\%) \\
\hline ESRD & $3(20 \%)$ \\
\hline Heart failure & $1(6.7 \%)$ \\
\hline Myocardial infarction & 0 \\
\hline \multicolumn{2}{|l|}{ Coronary revascularization } \\
\hline $\mathrm{CABG}$ & 0 \\
\hline $\mathrm{PCI}$ & $3(20 \%)$ \\
\hline Current tobacco use & $2(13.3 \%)$ \\
\hline \multicolumn{2}{|l|}{ SPECT MPI characteristics } \\
\hline Outpatient & $10(67 \%)$ \\
\hline $\begin{array}{l}\text { Days between first positive test } \\
\text { and MPI study }\end{array}$ & 65 days [31-94] \\
\hline Tested within $72 \mathrm{~h}$ before MPI & $9(60 \%)$ \\
\hline \multicolumn{2}{|l|}{ Type of study } \\
\hline Exercise & $4(27 \%)$ \\
\hline Duration & $\begin{array}{l}8.3 \text { minutes [6- } \\
10.2]\end{array}$ \\
\hline MET & $\begin{array}{l}\text { 10.7 METS } \\
{[8.4-11.7]}\end{array}$ \\
\hline Reported dyspnea & $3(75 \%)$ \\
\hline Regadenoson & $11(73 \%)$ \\
\hline Reported dyspnea & 7 (63.6\%) \\
\hline Aminophylline administered & 1 \\
\hline \multicolumn{2}{|l|}{ Indication for study } \\
\hline Chest pain & $7(46.7 \%)$ \\
\hline Shortness of breath & $3(20 \%)$ \\
\hline Heart failure & $2(13.3 \%)$ \\
\hline Pre-operative evaluation & $2(13.3 \%)$ \\
\hline Ventricular arrhythmia & $1(6.7 \%)$ \\
\hline
\end{tabular}

after tracer injection allowing for adequate imaging. Of the patients that underwent exercise, $75 \%$ reported nonlimiting shortness of breath and $25 \%$ mild chest pain.

\section{DISCUSSION}

This is the first report in the literature describing the experience of performing stress testing and MPI in patients who were previously hospitalized with severe COVID-19. Laboratories, including our own, have started to encounter patients who have recovered from COVID-19 and are presenting for stress testing due to various indications. In this manuscript, we report on 15 patients who recovered from severe COVID infections that required hospitalization and thereafter underwent stress testing with MPI. Pharmacologic studies were preferred over exercise to help control the spread of the pandemic, but by incorporating safety protocols, we were able to perform exercise stress testing on some patients. We encountered no serious adverse effects in any of the patients regardless of stress modality, and none required transfer to an emergency department or admission to the hospital. Hopefully, our experience will serve as a template for other laboratories who are faced with increased demand to perform MPI testing on patients who have recovered from COVID-19 and require evaluation for ischemic heart disease.

\section{NEW KNOWLEDGE GAINED}

As we progress through the pandemic, more patients that have recovered from COVID-19 will be referred for MPI. We describe our experience in performing stress testing with exercise and regadenoson in patients who have recently recovered from severe COVID-19-related hospitalizations. Further data are needed in this regard to reassure referring providers regarding recommending stress testing with MPI in these patients. A multi-center registry guided by ASNC will provide useful information in this regard.

\section{APPENDIX 1A}




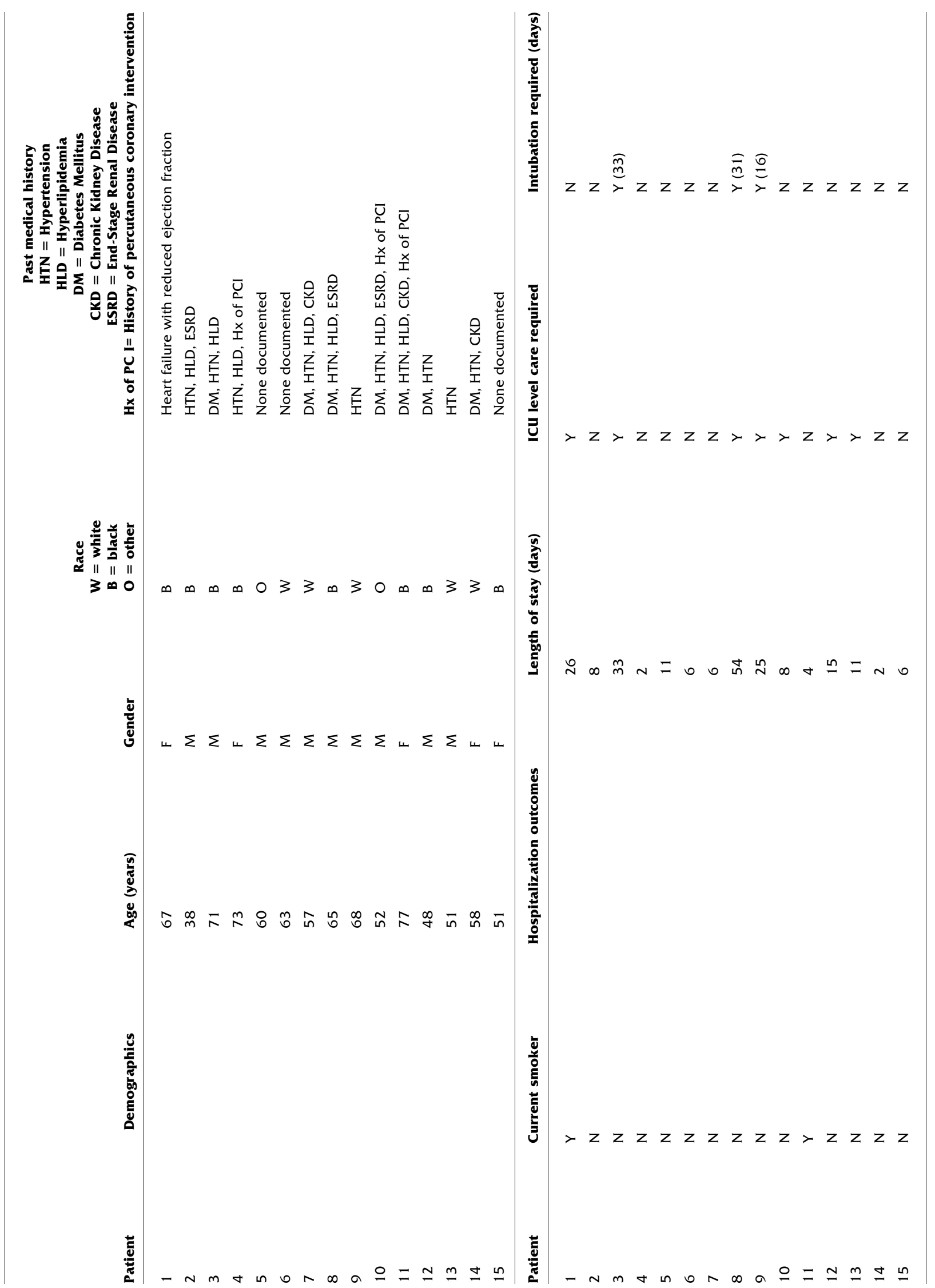




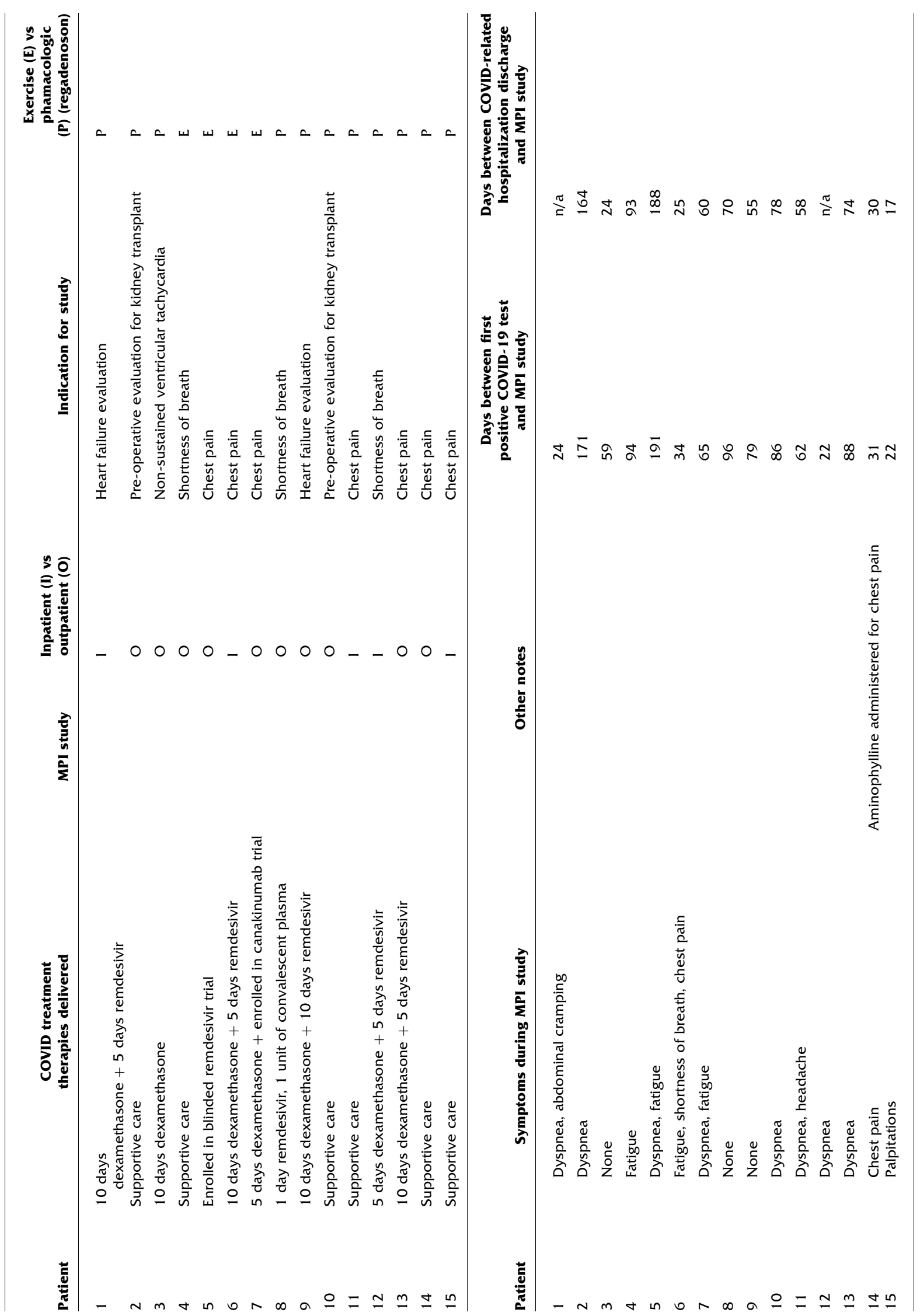




\section{APPENDIX A2}

\begin{tabular}{|c|c|c|c|c|c|c|c|c|c|c|c|}
\hline Patient & 1 & 2 & \multicolumn{4}{|c|}{3} & 4 & 5 & 6 & 7 & 8 \\
\hline \multicolumn{7}{|l|}{ Exercise duration } & 5 & 10.8 & 7 & 9.5 & \\
\hline \multicolumn{7}{|l|}{ METS } & 6.6 & 12 & 10 & 11 & \\
\hline SBP baseline & 126 & 129 & \multicolumn{4}{|c|}{152} & 104 & 145 & 135 & 137 & 131 \\
\hline DBP baseline & 91 & 70 & \multicolumn{4}{|c|}{89} & 73 & 82 & 87 & 84 & 77 \\
\hline HR peak & 118 & 112 & \multicolumn{4}{|c|}{103} & 146 & 163 & 143 & 148 & 98 \\
\hline \multicolumn{7}{|l|}{$\begin{array}{l}\% \text { maximum age-predicted HR } \\
\text { achieved }\end{array}$} & 99 & 101 & 90 & 90 & \\
\hline HR response $(\%)$ & 15 & 67 & \multicolumn{4}{|c|}{18} & & & & & 42 \\
\hline Perfusion defect & No & No & \multicolumn{4}{|c|}{$\begin{array}{l}\text { Y (scar, } 15 \% \text { of LV in LAD } \\
\text { distribution) }\end{array}$} & No & No & No & No & No \\
\hline LVEF (\%) & 38 & 28 & \multicolumn{4}{|c|}{49} & 64 & 51 & 71 & 65 & 61 \\
\hline \multicolumn{2}{|l|}{ Patient } & \multicolumn{2}{|r|}{9} & 10 & 11 & 12 & & 13 & 14 & & 15 \\
\hline \multicolumn{12}{|l|}{ Exercise duration } \\
\hline \multicolumn{12}{|l|}{ METS } \\
\hline \multicolumn{2}{|l|}{ SBP baseline } & & 125 & 189 & 143 & 141 & & 131 & 101 & & 94 \\
\hline \multicolumn{2}{|l|}{ DBP baseline } & & 79 & 92 & 79 & 90 & & 86 & 71 & & 60 \\
\hline \multicolumn{2}{|c|}{ HR peak } & & 85 & 87 & 96 & 115 & & 94 & 115 & & 135 \\
\hline \multicolumn{12}{|c|}{$\%$ maximum age-predicted $H R$ achieved } \\
\hline \multicolumn{2}{|l|}{ HR response (\%) } & & 37 & 14 & 35 & 22 & & 57 & 83 & & 35 \\
\hline \multicolumn{2}{|l|}{ Perfusion defect } & & No & No & No & No & & No & No & & No \\
\hline \multicolumn{2}{|l|}{ LVEF (\%) } & & 70 & 46 & 55 & 38 & & 56 & 60 & & 78 \\
\hline
\end{tabular}

\section{References}

1. Diaz A, Sarac BA, Schoenbrunner AR, Janis JE, Pawlik TM. Elective surgery in the time of COVID-19. Am J Surg 2020;219(6):900-2. https://doi.org/10.1016/j.amjsurg.2020.04.014.

2. Lancet The. COVID-19: protecting health-care workers. Lancet 2020;395(10228):922. https://doi.org/10.1016/S0140-6736(20)30 644-9.

3. Skulstad H, Cosyns B, Popescu BA, et al. COVID-19 pandemic and cardiac imaging: EACVI recommendations on precautions, indications, prioritization, and protection for patients and healthcare personnel. Eur Heart J Cardiovasc Imaging 2020;21(6):592-8. https://doi.org/10.1093/ehjci/jeaa072.

4. Naidich JJ, Boltyenkov A, Wang JJ, Chusid J, Hughes D, Sanelli PC. Impact of the coronavirus disease 2019 (COVID-19) pandemic on imaging case volumes. J Am Coll Radiol 2020;17(7):865-72. https://doi.org/10.1016/j.jacr.2020.05.004.

5. Hasnie UA, Bhambhvani P, Iskandrian AE, Hage FG. Prevalence of abnormal SPECT myocardial perfusion imaging during the COVID-19 pandemic [published online ahead of print, 2021 Jan 8]. Eur J Nucl Med Mol Imaging 2021. https://doi.org/10.1007/ s00259-020-05123-z.

6. Skali H, Murthy VL, Al-Mallah MH, et al. Guidance and best practices for nuclear cardiology laboratories during the coronavirus disease 2019 (COVID-19) pandemic: An Information Statement from ASNC and SNMMI. J Nucl Cardiol 2020;27(3):1022-9. https://doi.org/10.1007/s12350-020-02123-2.
7. Skali H, Murthy VL, Paez D, et al. Guidance and best practices for reestablishment of non-emergent care in nuclear cardiology laboratories during the coronavirus disease 2019 (COVID-19) pandemic: An information statement from ASNC, IAEA, and SNMMI : Endorsed by the Infectious Diseases Society of America [published online ahead of print, 2020 Jul 24]. J Nucl Cardiol. 2019. https://doi.org/10.1007/s12350-020-02203-3.

8. Carfì A, Bernabei R, Landi F. Gemelli against COVID-19 postacute care study group. Persistent symptoms in patients after acute COVID-19. JAMA 2020;324(6):603-5. https://doi.org/10.1001/ja ma.2020.12603.

9. Henzlova MJ, Cerqueira MD, Mahmarian JJ, Yao SS. Quality Assurance Committee of the American Society of Nuclear Cardiology. Stress protocols and tracers. J Nucl Cardiol 2006;13(6):e80-90. https://doi.org/10.1016/j.nuclcard.2006.08. 011.

10. Hage FG, Dean P, Iqbal F, Heo J, Iskandrian AE. A blunted heart rate response to regadenoson is an independent prognostic indicator in patients undergoing myocardial perfusion imaging. J Nucl Cardiol 2011;18(6):1086-94. https://doi.org/10.1007/s12350-0119429-1.

11. Hage FG, Ghimire G, Lester D, et al. The prognostic value of regadenoson myocardial perfusion imaging. J Nucl Cardiol 2015;22(6):1214-21. https://doi.org/10.1007/s12350-014-0050-y.

12. Ives CW, AlJaroudi WA, Kumar V, et al. Prognostic value of myocardial perfusion imaging performed pre-renal transplantation: post-transplantation follow-up and outcomes. Eur J Nucl Med Mol 
Imaging 2018;45(11):1998-2008. https://doi.org/10.1007/s00259018-4068-2.

13. Colon CM, Marshell RL, Roth CP, Farag AA, Iskandrian AE, Hage FG. The prognostic value of myocardial perfusion imaging in patients with type 2 myocardial infarction [published online ahead of print, 2019 Oct 23]. J Nucl Cardiol 2019. https://doi.org/ 10.1007/s12350-019-01915-5.

14. McRee CW, Brice LR, Farag AA, Iskandrian AE, Hage FG. Evolution of symptoms in patients with stable angina after normal regadenoson myocardial perfusion imaging: The Radionuclide Imaging and Symptomatic Evolution study (RISE). J Nucl Cardiol 2020. https://doi.org/10.1007/s12350-020-02298-8.

Publisher's Note Springer Nature remains neutral with regard to jurisdictional claims in published maps and institutional affiliations. 